Pacific

Journal of

Mathematics

\title{
THE CONSTANT OF INTERPOLATION
}

Artur Nicolau, Joaquim Ortega-Cerdà, and Kristian Seip 


\title{
THE CONSTANT OF INTERPOLATION
}

\author{
Artur Nicolau, Joaquim Ortega-Cerdà, and Kristian Seip
}

\begin{abstract}
We prove that a suitably adjusted version of Peter Jones' formula for interpolation in $H^{\infty}$ gives a sharp upper bound for what is known as the constant of interpolation. We show how this leads to precise and computable numerical bounds for this constant.
\end{abstract}

With each finite or infinite sequence $Z=\left(z_{j}\right)(j=1,2, \ldots)$ of distinct points $z_{j}=x_{j}+i y_{j}$ in the upper half-plane of the complex plane, we associate a number $M(Z) \in \mathbb{R}^{+} \cup\{+\infty\}$ which we call the constant of interpolation. We may define it in two equivalent ways. The first is related to Carleson's interpolation theorem for $H^{\infty}$ [Car58]. We say that $Z$ is an interpolating sequence if the interpolation problem

$$
f\left(z_{j}\right)=w_{j}, j=1,2, \ldots
$$

has a solution $f \in H^{\infty}$ for each bounded sequence $\left(w_{j}\right)$ of complex numbers. Using the open mapping theorem, we find that if $Z$ is an interpolating sequence, then we can always solve (1) with a function $f$ such that

$$
\|f\|_{\infty} \leq C\left\|\left(w_{j}\right)\right\|_{\infty}
$$

for some $C<\infty$ depending only on $Z$. The constant of interpolation $M(Z)$ is declared to be the smallest such $C$. We set $M(Z)=+\infty$ if $Z$ is not an interpolating sequence.

By a classical theorem of Pick (see [Gar81, p. 2]), we may alternatively define $M(Z)$ as follows: Let $M_{n}(Z)$ be the smallest number $C$ such that the matrices

$$
\left(\frac{1-\overline{w_{j}} w_{k}}{z_{j}-\overline{z_{k}}}\right)_{j, k=1,2, \ldots, n}
$$

are positive semi-definite whenever $\left\|\left(w_{j}\right)\right\|_{\infty} \leq 1 / C$. The constant of interpolation is then $M(Z)=M_{n}(Z)$ if $Z$ is a finite sequence consisting of $n$ points and $M(Z)=\lim _{n \rightarrow \infty} M_{n}(Z)$ if $Z$ is infinite. We will make no use of this definition, but have stated it to make the reader aware of the relevance of $M(Z)$ for the classical Nevanlinna-Pick problem. Note that this connection has been investigated by Koosis, who derived Carleson's theorem formally from Pick's theorem [Koo00]. 
Carleson's interpolation theorem [Car58] states that $Z$ is an interpolating sequence (or alternatively $M(Z)<\infty$ ) if and only if

$$
\delta(Z)=\inf _{j \neq k} \prod_{k \neq j}\left|\frac{z_{j}-z_{k}}{z_{j}-\overline{z_{k}}}\right|>0 .
$$

Clearly, an interpolating sequence satisfies the Blaschke condition. We let $B$ be the associated Blaschke product and set

$$
B_{j}(z)=\frac{z-\overline{z_{j}}}{z-z_{j}} B(z)
$$

so that we may write $\delta(Z)=\inf _{j}\left|B_{j}\left(z_{j}\right)\right|$.

An interesting result related to Carleson's theorem is that if $M(Z)<\infty$, then the interpolation may be obtained by means of a linear operator. In fact, P. Beurling [Car63] proved that there exist $f_{j} \in H^{\infty}$ with $f_{j}\left(z_{j}\right)=1$ and $f_{j}\left(z_{k}\right)=0$ if $k \neq j$, such that

$$
M(Z)=\sup _{z} \sum_{j}\left|f_{j}(z)\right| .
$$

The functions $f_{j}$ have the form

$$
f_{j}(z)=\frac{B_{j}(z)}{B_{j}\left(z_{j}\right)}\left(\frac{2 i y_{j}}{z-\overline{z_{j}}}\right)^{2} \frac{G\left(z_{j}\right)}{G(z)},
$$

where $G$ is a bounded analytic function solving a certain nonlinear extremal problem. Unfortunately, $G$ is not given explicitly, and it seems very difficult to get much further. The problem of finding $G$ can be seen as a version of the Nevanlinna-Pick interpolation problem, where one is interested in computing $M(Z)$ and finding solutions of minimal norm. There are classical results of R. Nevanlinna describing these solutions, but they are very implicit and give little help in concrete situations. It is therefore of interest to find more explicit solution operators, along with good estimates for $M(Z)$.

A remarkably simple formula was found by P. Jones [Jon83]. He showed that the series

$$
f(z)=\sum_{j} w_{j} \frac{B_{j}(z)}{B_{j}\left(z_{j}\right)}\left(\frac{2 i y_{j}}{z-\overline{z_{j}}}\right)^{2} \exp \left(-a i \sum_{y_{k} \leq y_{j}}\left(\frac{y_{k}}{z-\overline{z_{k}}}-\frac{y_{k}}{z_{j}-\overline{z_{k}}}\right)\right)
$$

defines a function $f \in H^{\infty}$ such that $f\left(z_{j}\right)=w_{j}$ with $\|f\| \leq C\left\|\left(w_{j}\right)\right\|_{\infty}$. Here $a$ can be chosen freely and $C$ is a constant depending on $a$ and the sequence $Z$. Jones' formula can be tweaked in several ways. For instance, in [Vin83], Vinogradov found functions $f_{j}$ that are rational when the interpolating sequence is finite. This is of interest for some applications; see [Nik02, p. 179]. The purpose of this note is to show that this explicit operator, conveniently adjusted, is close to optimal. By considering a certain extreme configuration of points, we are in fact able to prove that it yields 
a sharp upper bound for $M(Z)$. As a result, $M(Z)$ may be bounded from above and below by fairly explicit numerical constants.

We begin by showing how to "optimize" Jones' formula. Take an analytic function $g$ such that $g(i)=1$. We need $|g|$ to have a harmonic majorant, so we require $(z+i)^{-2} g(z) \in H^{1}$ [Gar81, p. 60]. Let $u$ denote the least harmonic majorant of $|g|$ and set

$$
g_{j}(z)=g\left(\left(z-x_{j}\right) / y_{j}\right), \quad u_{j}(z)=u\left(\left(z-x_{j}\right) / y_{j}\right) .
$$

We assume further that $g$ is such that

$$
U_{k}(z)=\sum_{y_{j} \leq y_{k}} \frac{u_{j}(z)}{\left|B_{j}\left(z_{j}\right)\right|}
$$

defines a harmonic function; let $V_{k}(z)$ be a harmonic conjugate of $U_{k}$, and set $G_{k}=U_{k}+i V_{k}$. This leads us to the following interpolation formula:

$$
f(z)=\sum_{j} w_{j} \frac{B_{j}(z)}{B_{j}\left(z_{j}\right)} g_{j}(z) \exp \left(-a\left(G_{j}(z)-G_{j}\left(z_{j}\right)\right)\right)
$$

with $a$ some constant which may be chosen freely. Clearly, $f\left(z_{j}\right)=w_{j}$. We define

$$
c_{J}(Z, g)=\sup _{j} U_{j}\left(z_{j}\right)
$$

so that for arbitrary $z$ we get the estimate

$$
|f(z)| \leq\left\|\left(w_{j}\right)\right\|_{\infty} \frac{\exp \left(a c_{J}(Z, g)\right)}{a} \sum_{j} \frac{a\left|g_{j}(z)\right|}{\left|B_{j}\left(z_{j}\right)\right|} \exp \left(-a U_{j}(z)\right) .
$$

Replacing $\left|g_{j}\right|$ by $u_{j}$, we find that the latter sum is a lower Riemann sum for the integral

$$
\int_{0}^{\infty} e^{-t} d t
$$

so that we arrive at the estimate

$$
|f(z)| \leq\left\|\left(w_{j}\right)\right\|_{\infty} \frac{\exp \left(a c_{J}(Z, g)\right)}{a} .
$$

We see that the optimal choice of $a$ is $1 / c_{J}(Z, g)$, and this leads us to the bound

$$
M(Z) \leq e c_{J}(Z, g) .
$$

We may finally minimize $c_{J}(Z, g)$ and define

$$
c_{J}(Z)=\inf _{g} c_{J}(Z, g)
$$

so that

$$
M(Z) \leq e c_{J}(Z) .
$$


We have then proved one part of the following theorem:

Theorem 1. For every sequence $Z$ in the upper half-plane,

$$
M(Z) \leq e c_{J}(Z)
$$

The inequality is best possible in the sense that the constant e on the right side of (2) cannot be replaced by any smaller number.

We postpone for the moment the proof of the sharpness of (2); it will be established by means of an explicit example at the end of this note.

It may be argued that finding the $g$ minimizing $c_{J}(Z, g)$ is not much easier than solving for the function $G$ in P. Beurling's formula. However, we will now point out that $c_{J}(Z)$ relates nicely to more computable characteristics.

An immediate observation is that if we choose $g(z)=-4 /(z+i)^{2}$, then $u(z)=4(y+1) /|z+i|^{2}$ so that $c_{J}(Z, g)$ becomes

$$
c_{H J}(Z)=\sup _{n} \sum_{y_{j} \leq y_{n}} \frac{4 y_{j}\left(y_{j}+y_{n}\right)}{\left|z_{j}-\overline{z_{n}}\right|^{2}} \frac{1}{\left|B_{j}\left(z_{j}\right)\right|} .
$$

This choice of $g$ corresponds to the original version of Jones' formula. (The letter ' $H$ ' in $c_{H J}(Z)$ stands for Havin; see below.) For this characteristic we have the following result:

Theorem 2. For every sequence $Z$ in the upper half-plane,

$$
M(Z) \leq k c_{H J}(Z)
$$

for some universal constant $k$. The best possible $k$ lies in the interval $[\pi / \log 4, e]=[2.2662 \ldots, 2.7183 \ldots]$.

We have already established the upper bound for $k$. The lower bound will again follow from the example to be considered below.

Our third and final characteristic was introduced by V. Havin in the first appendix of [Koo98]. Havin's presentation in [Koo98] was based on work by Vinogradov, Gorin, and Hruščëv [VGH81]. We get Havin's characteristic from the expression for $M(Z)$ obtained from Carleson's duality argument (see [Gar81, p. 135]):

$$
M(Z)=\sup \left\{4 \pi \sum \frac{y_{j}\left|h\left(z_{j}\right)\right|}{\left|B_{j}\left(z_{j}\right)\right|}: h \in H^{1},\|h\|_{1} \leq 1\right\} .
$$

If we choose $h(z)=\pi^{-1} y_{k} /\left(z-\overline{z_{k}}\right)^{2}, k=1,2, \ldots$, we arrive at

$$
c_{H}(Z)=\sup _{k} \sum_{j} \frac{4 y_{k} y_{j}}{\left|z_{k}-\bar{z}_{j}\right|^{2}} \frac{1}{\left|B_{j}\left(z_{j}\right)\right|}
$$

along with the estimate

$$
M(Z) \geq c_{H}(Z)
$$


Since clearly $c_{H J}(Z) \leq 2 c_{H}(Z)$, we may summarize our findings as a chain of inequalities:

$$
c_{H}(Z) \leq M(Z) \leq e c_{J}(Z) \leq e c_{H J}(Z) \leq 2 e c_{H}(Z) .
$$

In [Koo98], Havin proves that

$$
c_{H}(Z) \leq M(Z) \leq k c_{H}(Z),
$$

with $k$ a universal constant. To prove the right inequality, he proceeds by duality and uses the invariant Blaschke characterization of Carleson measures, which is closely related to the original proof of Carleson. By computing both $c_{H}(Z)$ and $M(Z)$ when $Z$ consists of two points, he also shows that the left inequality is best possible. In fact, it may be checked that each of the inequalities in our chain (3) is sharp.

To interpret the "geometric" contents of our characteristics, it may be useful to relate them to the condition

$$
\sup _{k} \sum_{j} \frac{y_{j} y_{k}}{\left|z_{k}-\bar{z}_{j}\right|^{2}}<+\infty
$$

which is called the invariant Blaschke condition (see [Gar81, p. 239]). We see that our three characteristics are closely related to the supremum appearing in (4). It may also be noted that by the bound $M(Z) \leq 2 e c_{H}(Z)$ and a calculus argument applied to the invariant Blaschke sum, we obtain

$$
M(Z) \leq \frac{2 e+4 e \log (1 / \delta(Z))}{\delta(Z)}
$$

see $[$ Koo98, p. 268].

We finally turn to our example which proves the sharpness of (2) and the lower bound for $k$ in Theorem 2. In what follows the notation $a(\gamma) \sim b(\gamma)$ will mean that $a(\gamma)$ and $b(\gamma)$ are asymptotically equal, i.e.,

$$
\lim _{\gamma \rightarrow+\infty} a(\gamma) / b(\gamma)=1
$$

An example. Fix $\gamma>0$ and consider the Blaschke product defined by

$$
B(z)=B(\gamma, z)=\prod_{k \leq 0} \frac{z-i e^{k / \gamma}}{z+i e^{k / \gamma}} \prod_{k>0} \frac{i e^{k / \gamma}-z}{z+i e^{k / \gamma}} .
$$

The signs have been chosen so that $i B^{\prime}(i)>0$, which ensures convergence of the product. The sequence of zeros $Z_{\gamma}=\left(i e^{k / \gamma}\right)_{k \in \mathbb{Z}}$ is clearly an interpolating sequence with $M\left(Z_{\gamma}\right)$ blowing up when $\gamma$ tends to $+\infty$. To obtain appropriate estimates for $B$, we relate it to the function

$$
F(z)=2 e^{-\frac{\pi^{2} \gamma}{2}} \sin (\pi \gamma \log (-i z)),
$$

where $\log (z)$ is the principal branch of the logarithm. Both $B$ and $F$ are bounded functions, and they have the same zeros. The quotient $F(z) / B(z)$ 
is an outer function with modulus close to 1 when $\gamma$ is large. More precisely, we have

$$
\sup _{x \in \mathbb{R} \backslash\{0\}}\left|\log \frac{|F(x)|}{|B(x)|}\right| \sim e^{-\pi^{2} \gamma},
$$

and therefore the same asymptotic relation holds in the upper half-plane. The Blaschke product $B$ is highly symmetric. It is real on the imaginary half-axis $i \mathbb{R}^{+}$and moreover $B\left(e^{1 / \gamma} z\right)=-B(z)$. We check that on $i \mathbb{R}^{+}$the modulus of $B$ peaks at the points $\left\{i e^{(k+1 / 2) / \gamma}: k \in \mathbb{Z}\right\}$. Again comparing it to $F$, we check that

$$
B\left(i e^{(k+1 / 2) / \gamma}\right)=(-1)^{k} 2 e^{-\frac{\pi^{2} \gamma}{2}} t_{\gamma} \text { with } t_{\gamma} \sim 1
$$

We will now obtain a lower estimate for $M\left(Z_{\gamma}\right)$ by finding a minimal norm solution of the interpolation problem

$$
f\left(i e^{k / \gamma}\right)=(-1)^{k}, k \in \mathbb{Z}
$$

By (5), the problem is solved by the function

$$
g(z)=c_{\gamma} B\left(e^{1 /(2 \gamma)} z\right)
$$

with $c_{\gamma}$ an appropriate constant satisfying $c_{\gamma} \sim e^{\frac{\pi^{2} \gamma}{2}} / 2$. This means that if we can prove that $g$ is a minimal norm solution, then it follows that

$$
M\left(Z_{\gamma}\right) \geq \frac{t_{\gamma}}{2} e^{\frac{\pi^{2} \gamma}{2}} \text { with } t_{\gamma} \sim 1
$$

We wish to prove that $g$ is a solution of minimal norm. To this end, observe that an arbitrary minimal norm solution can expressed as

$$
f=g+h B
$$

with $h$ a bounded analytic function. We may assume that $f$ is real on $i \mathbb{R}^{+}$ because by symmetry we may if necessary replace $f$ by $(\overline{f(-\bar{z})}+f(z)) / 2$. Thus $h$ is also real on $i \mathbb{R}^{+}$. We define

$$
h_{m}(z)=\frac{1}{m} \sum_{k=0}^{m-1} h\left(e^{2 k / \gamma} z\right),
$$

and choose a convergent subsequence $h_{m_{k}}(z) \rightarrow \widetilde{h}(z)$ such that the limit function satisfies $\widetilde{h}\left(e^{2 / \gamma} z\right)=\widetilde{h}(z)$, and $\widetilde{h}(i y) \in \mathbb{R}$ for real $y$. Hence $\widetilde{f}=g+\widetilde{h} B$ is also a minimal norm solution and $\widetilde{f}\left(e^{2 / \gamma} z\right)=f(z)$. Finally, note that

$$
\varphi(z)=\frac{1}{2}\left(\widetilde{f}(z)-\widetilde{f}\left(e^{1 / \gamma} z\right)\right)
$$

is a minimal norm solution as well such that

$$
\varphi\left(e^{1 / \gamma} z\right)=-\varphi(z)
$$


Assume now that $g$ is not a minimal norm solution. Then $\|\varphi\|_{\infty}<\|g\|_{\infty}$. Between the points $i$ and $i e^{1 / \gamma}, \varphi$ has a zero $i \delta$ because it is real on $i \mathbb{R}$. Therefore, by the periodicity expressed by (7), $\varphi$ has zeros at $i \delta e^{k / \gamma}, k \in \mathbb{Z}$. It follows that we may factorize $\varphi$ as

$$
\varphi(z)=B(z / \delta) \varphi_{0}(z) .
$$

We evaluate $\varphi$ at the point $i$ and get

$$
1=|\varphi(i)|=\left|B(i / \delta)\left\|\varphi_{0}(i) \mid \leq \frac{1}{c_{\gamma}}\right\| \varphi_{0} \|_{\infty}=\frac{\|\varphi\|_{\infty}}{c_{\gamma}}=\frac{\|\varphi\|_{\infty}}{\|g\|_{\infty}}<1,\right.
$$

which is a contradiction. We conclude that $g$ has minimal norm so that (6) holds.

The next step is to compute $c_{J}\left(Z_{\gamma}\right)$. Since $B\left(e^{1 / \gamma} z\right)=-B(z)$, we have that

$$
\left|e^{k / \gamma} B^{\prime}\left(i e^{k / \gamma}\right)\right|=\left|B^{\prime}(i)\right|
$$

for each integer $k$. Hence

$$
\left|B_{k}\left(i e^{k / \gamma}\right)\right|=2 e^{k / \gamma}\left|B^{\prime}\left(i e^{k / \gamma}\right)\right|=2\left|B^{\prime}(i)\right| .
$$

The derivative $B^{\prime}(i)$ can be estimated in terms of $F^{\prime}(i)$, which gives us

$$
i B^{\prime}(i) e^{\frac{\pi^{2} \gamma}{2}} /(2 \pi \gamma) \rightarrow 1 \text { as } \gamma \rightarrow+\infty \text {. }
$$

Thus

$$
c_{J}\left(Z_{\gamma}\right) \sim(4 \pi \gamma)^{-1} e^{\frac{\pi^{2} \gamma}{2}} \inf _{g(i)=1} \sup _{k \in \mathbb{Z}} \sum_{y_{j} \leq y_{k}} u\left(i y_{k} / y_{j}\right)
$$

with $u$ denoting as before the least harmonic majorant of $|g|$. Using the explicit expression for this majorant, we get

$$
\inf _{g(i)=1} \sup _{k \in \mathbb{Z}} \sum_{y_{j} \leq y_{k}} u\left(i y_{k} / y_{j}\right)=\inf _{g(i)=1} \sum_{k \geq 0} \frac{1}{\pi} \int_{\mathbb{R}} \frac{1}{1+t^{2}}\left|g\left(e^{k / \gamma} t\right)\right| d t .
$$

We interpret the sum on the right as a Riemann sum, so that

$$
\begin{aligned}
\sum_{y_{j} \leq y_{k}} u\left(i y_{k} / y_{j}\right) & \sim \frac{\gamma}{\pi} \int_{\mathbb{R}} \frac{1}{1+t^{2}} \int_{0}^{\infty}\left|g\left(t e^{x}\right)\right| d x d t \\
& =\frac{\gamma}{\pi} \int_{\mathbb{R}} \frac{1}{1+t^{2}} \int_{t}^{\infty} \frac{|g(u)|}{u} d u d t .
\end{aligned}
$$

Integrating by parts, we get

$$
\sum_{y_{j} \leq y_{k}} u\left(i y_{k} / y_{j}\right) \sim \frac{\gamma}{\pi} \int_{\mathbb{R}} \frac{\arctan t}{t}|g(t)| d t
$$


We want to minimize the latter integral over all functions $g$ such that $(z+$ $i)^{-1} g \in H^{1}$ and $g(i)=1$. This can be restated as an extremal problem in the weighted Hardy space with norm

$$
\|h\|^{2}=\int_{\mathbb{R}}|h(t)|^{2} \frac{\arctan t}{t} d t .
$$

In turn, we can reduce this problem to one for the standard Hardy space $H^{2}$, and we find that our original problem is solved by the function

$$
g_{0}(z)=\left(\frac{2 i}{z+i}\right)^{2} \frac{\psi(z)}{\psi(i)},
$$

where $\psi(z)$ is the outer function whose modulus is $t / \arctan t$ on $\mathbb{R}$. Since

$$
\int_{\mathbb{R}}\left|g_{0}(t)\right| \frac{\arctan t}{t} d t=\int_{\mathbb{R}} \frac{4}{\left(t^{2}+1\right)} \frac{1}{|\psi(i)|}=\frac{4 \pi}{|\psi(i)|},
$$

we get

$$
c_{J}\left(Z_{\gamma}\right) \sim \frac{e^{\frac{\pi^{2} \gamma}{2}}}{\pi|\psi(i)|}
$$

when plugging our extremal function $g_{0}$ into (8).

We are left with the computation of $|\psi(i)|$. We first note that

$$
\psi(i)=i \exp \left(-\frac{i}{\pi} \int_{\mathbb{R}}\left(\frac{1}{i-t}+\frac{t}{t^{2}+1}\right) \log |\arctan (t)| d t\right) .
$$

Since

$$
|\arctan (t)|=\frac{1}{2}\left|\log \left(\frac{1-i t}{1+i t}\right)\right|,
$$

the change of variables

$$
e^{i \theta}=\frac{1-i t}{1+i t}
$$

brings us to the explicit expression

$$
\psi(i)=2 i \exp \left(-\frac{1}{2 \pi} \int_{-\pi}^{\pi} \log \left|\log e^{i \theta}\right| d \theta\right)=\frac{2 i e}{\pi} .
$$

Combining (6) and (9), we conclude that

$$
c_{J}\left(Z_{\gamma}\right) \sim \frac{1}{2 e} e^{\frac{\pi^{2} \gamma}{2}} \leq \frac{t_{\gamma}}{e} M\left(Z_{\gamma}\right) \text { with } t_{\gamma} \sim 1,
$$

which proves the sharpness of (2) of Theorem 1.

The computation of $c_{H J}\left(Z_{\gamma}\right)$ is straightforward. Indeed,

$$
c_{H J}\left(Z_{\gamma}\right) \sim(4 \pi \gamma)^{-1} e^{\frac{\pi^{2} \gamma}{2}} \sum_{k \geq 0} \frac{4 e^{-k / \gamma}}{\left(1+e^{-k / \gamma}\right)} .
$$


The sum is again regarded as a Riemann sum, i.e.,

$$
\sum_{k \geq 0} \frac{e^{-k / \gamma}}{1+e^{-k / \gamma}} \sim \gamma \int_{0}^{\infty} \frac{e^{-x}}{1+e^{-x}} d x=\gamma \log 2
$$

so that we arrive at the relation

$$
c_{H J}\left(Z_{\gamma}\right) \sim \frac{\log 2}{\pi} e^{\frac{\pi^{2} \gamma}{2}} \leq \frac{t_{\gamma} 2 \log 2}{\pi} M\left(Z_{\gamma}\right) \text { with } t_{\gamma} \sim 1
$$

This proves the lower bound for $k$ in Theorem 2 .

Acknowledgments. . We are grateful to N. Nikolski for communicating to us several relevant remarks on the bibliography of this paper.

\section{References}

[Car58] L. Carleson, An interpolation problem for bounded analytic functions, Amer. J. Math., 80 (1958), 921-930, MR 22 \#8129, Zbl 0085.06504.

[Car63] - Interpolations by bounded analytic functions and the Corona problem, Proc. Internat. Congr. Mathematicians (Stockholm, 1962), Inst. Mittag-Leffler, Djursholm, 1963, 314-316, MR 31 \#549, Zbl 0192.16801.

[Gar81] J.B. Garnett, Bounded Analytic Functions, Pure and Applied Mathematics, 96, Academic Press Inc. [Harcourt Brace Jovanovich Publishers], New York, 1981, MR 83g:30037, Zbl 0469.30024.

[Jon83] P.W. Jones, $L^{\infty}$ estimates for the $\bar{\partial}$ problem in a half-plane, Acta Math., 150(12) (1983), 137-152, MR 84g:35135, Zbl 0516.35060.

[Koo98] P. Koosis, Introduction to $H_{p}$ Spaces, second ed., Cambridge Tracts in Mathematics, 115, Cambridge University Press, Cambridge, 1998, with two appendices by V.P. Havin [Viktor Petrovich Khavin], MR 2000b:30052.

[Koo00] P. Koosis, Carleson's interpolation theorem deduced from a result of Pick, Complex Analysis, Operators, and Related Topics, Oper. Theory Adv. Appl., 113, Birkhäuser, Basel, 2000, 151-162. MR 2002a:30061 Zbl 0976.30021.

[Nik02] N. K. Nikolski, Operators, Functions, and Systems: An Easy Reading, Vol. 2, Mathematical Surveys and Monographs, 93, American Mathematical Society, Providence, RI, 2002, Model operators and systems, Translated from the French by Andreas Hartmann and revised by the author. CMP 1892 647, Zbl 1007.47002.

[Vin83] S. A. Vinogradov, Some remarks on free interpolation by bounded and slowly growing analytic functions, Zap. Nauchn. Sem. Leningrad. Otdel. Mat. Inst. Steklov. (LOMI), 126 (1983), 35-46, Investigations on linear operators and the theory of functions, XII. MR 85d:30053, Zbl 0515.30025.

[VGH81] S. A. Vinogradov, E. A. Gorin, and S. V. Hruščëv, Free interpolation in $H^{\infty}$ in the sense of P. Jones, Zap. Nauchn. Sem. Leningrad. Otdel. Mat. Inst. Steklov. (LOMI), 113 (1981), 212-214, 268, Investigations on linear operators and the theory of functions, XI. MR 82k:46039, Zbl 0515.30026.

Received January 27, 2003. The authors are supported by the European Commission Research Training Network HPRN-CT-2000-00116. The first two authors are supported by DGICYT grants: BFM2002-00571, BFM2002-04072-C02-01 and by the CIRIT: 
2001SGR00172, 2001SGR00431. The third author is partly supported by a grant from the Research Council of Norway.

Dept. Matemàtiques

Universitat Autònoma de Barcelona

08193 BeLlaterRA

SPAIN

E-mail address: artur@mat.uab.es

Dept. Matemàtica Aplicada i AnÀlisi

UNIVERSitAT DE BARCELONA

Gran Via 585

08071 BARCELONA

SPAIN

E-mail address: quim@mat.ub.es

Dept. of Mathematical Sciences

Norwegian University of Science and Technology

N-7491 TrondHEIM

NORWAY

E-mail address: seip@math.ntnu.no 